\title{
Design and Deployment of Visible-Thermal Biometric Surveillance Systems
}

\author{
Diego A. Socolinsky \\ Equinox Corporation \\ 207 E. Redwood St \\ Baltimore, MD 21202 \\ diegodequinoxsensors.com
}

Automatic video surveillance in uncontrolled outdoor settings is a very challenging computer vision task. Nearly infinite variability of the environmental factors and the open-ended goals of many surveillance problems conspire to create situations where even the most advanced detection, tracking and recognition algorithms falter. While the common academic response to such challenges is to develop new, more powerful algorithms capable of handling a broader range of conditions with acceptable performance, this course of action is sometimes not appropriate from the industrial-commercial point of view. Sometimes systems must be deployed sooner than would allow for the development cycle of complex new algorithms, and must be more robust than most such algorithms can be expected to be on short notice. Under those circumstances, one may look toward better data quality as one means of improving performance while remaining close to the existing state-of-the-art in algorithmic technology. This is often the motivation for deployment of multimodal surveillance systems in the real world.

Data quality can be quantified in many ways, but intuitively refers to the amount of relevant information conveyed by the data. As far as video surveillance is concerned, numerical gauges such as signal-to-noise ratio (SNR), pixel resolution, sensitivity and frame rate are common indicators of image quality that can be used as predictors of algorithmic performance. The joint use of multiple imaging modalities is one means of improving some of the quality measures of the input data, in hopes of ultimately improving overall system performance. For example, if the relevant task is detection of moving objects throughout the full diurnal cycle, then the addition of a low-light capable sensor to an existing standard visible surveillance camera will increase the system SNR during nighttime, and thus presumably improve overall detection performance. However, the complexity of the system is also increased by adding a second sensor, not to mention its cost. Is the addition of a low-light camera necessary, or could we instead replace the visible camera outright, and use the low-light sensor alone?
Would a different sensor technology be better altogether? What is the added performance versus the added cost for different sensor configurations? Will the choice of sensors have an impact if the relevant task changes to include object recognition in addition to detection? Our purpose is to address some of these questions from a practical point of view.

Since surveillance often requires operation in daytime and nighttime conditions alike, we will focus specifically on bi-modal systems in which at least one sensor is capable of imaging in the dark. This will lead us naturally to the combination of thermal infrared imagery with a purely reflective modality, such as standard visible imagery. The examples below are based on the author's experience designing, deploying and exploiting multimodal imaging systems. As such, they should not be taken as prescriptive, but rather as one practitioner's point of view.

The use of visible (or NIR) and thermal sensors in combination opens up new possibilities for surveillance applications. It allows us to transfer tried and true techniques which work in favorable illumination conditions to realistic outdoor scenarios. By leveraging the relative strengths of reflective and emmisive modalities, fused sensor systems are often capable of operating throughout the diurnal cycle. Their main advantage is the robustness to environmental variation, which ultimately results in higher performance. The success of any such deployment ultimately depends not only on performance, but also reliability and cost. These three factors are intimately linked to the design of the sensor platform itself, which in turn dictates the possibly algorithmic avenues. Proper design is critical to customer acceptance, and in the end has a major influence on the likelihood of deployment.

Real-world deployment is often dependent on measurably acceptable performance for a given task. It is very important to realize that properly defining the task has great influence in perceived and measured performance, and ultimately may mean the difference between an academic exercise and a commercially deployed system. For the case 
of visible-thermal biometric surveillance, we showed that despite the fact that visible-thermal face recognition can be academically shown to outperform visible alone, if the task is ill-suited to the technology, acceptable performance cannot be achieved. On the other hand, a properly selected task will greatly benefit from the added performance afforded by a visible-thermal system. This reasoning is applicable to many current aspects of biometric surveillance, both unimodal or multimodal. Unless the application realm is properly delimited, performance cannot be expected to be adequate, regardless of algorithmic or hardware progress. It will be of great benefit to the field to concentrate on achieving excellent results in feasible yet relevant scenarios, rather than mediocre ones in infeasible ones.

This presentation constitutes a brief foray into design issues for visible-thermal systems, with emphasis on biometric surveillance. We have purposely kept the exposition at a relatively high level, so as not to be mired by details. In this fashion, it will hopefully prove a useful introduction for the uninitiated and a subject of fruitful discussion for the more seasoned practitioner.

\section{References}

[1] D.A. Socolinsky and A. Selinger, "Face recognition with visible and thermal infrared imagery," Computer Vision and Image Understanding, July - August 2003.

[2] D. Socolinsky, L. Wolff, J. Neuheisel, and C. Eveland, "Illumination Invariant Face Recognition Using Thermal Infrared Imagery," in Proceedings CVPR, Kauai, Dec. 2001.

[3] F. J. Prokoski, "History, Current Status, and Future of Infrared Identification," in Proceedings IEEE Workshop on Computer Vision Beyond the Visible Spectrum: Methods and Applications, Hilton Head, 2000.

[4] D. A. Socolinsky and A. Selinger, "A comparative analysis of face recognition performance with visible and thermal infrared imagery," in Proceedings ICPR, Quebec, Canada, August 2002.

[5] L.B. Wolff, D.A. Socolinsky, and C.K. Eveland, "Face recognition in the thermal infrared," in Computer Vision Beyond the Visible Spectrum, I. Pavlidis and B. Bhanu, Eds. Springer Verlag, 2004.

[6] X. Chen, P. Flynn, and K. Bowyer, "Visible-light and infrared face recognition," in Proceedings of the Workshop on Multimodal User Authentication, Santa Barbara, CA, December 2003, to appear.

[7] X. Chen, P. Flynn, and K. Bowyer, "PCA-based face recognition in infrared imagery: Baseline and comparative studies," in International Workshop on Analysis and Modeling of Faces and Gestures, Nice, France, October 2003.

[8] R. Cutler, "Face recognition using infrared images and eigenfaces," Tech. Rep., University of Maryland, April 1996, Available at http://www.cs.umd.edu/ rgc/pub/ireigenface.pdf.

[9] Joseph Wilder, P. Jonathon Phillips, Cunhong Jiang, and Stephen Wiener, "Comparison of Visible and Infra-Red Imagery for Face Recognition," in Proceedings of 2nd International Conference on Automatic Face \& Gesture Recognition, Killington, VT, 1996, pp. 182-187.

[10] L. Wolff, D. Socolinsky, and C. Eveland, "Quantitative Measurement of Illumination Invariance for Face Recognition Using Thermal Infrared Imagery," in Proceedings CVBVS, Kauai, Dec. 2001.

[11] P. Buddharaju, I.T. Pavlidis, and P. Tsiamyrtzis, "Pose-invariant physiological face recognition in the thermal infrared spectrum," in Biometrics06, 2006, p. 53.

[12] T. Sakai, M. Nagao, and T. Kanade, "Computer analysis and classification of photographs of human faces," in Proc. First USA-JAPAN Computer Conference, 1972, pp. 55-62.

[13] D. A. Socolinsky and A. Selinger, "Thermal face recognition in an operational scenario," in Proceedings of CVPR 2004, Washington, D.C., 2004. 